Check for updates

Cite this: RSC Adv., 2017, 7, 38965

Received 7th June 2017

Accepted 2nd August 2017

DOI: 10.1039/c7ra06351b

rsc.li/rsc-advances

\section{Development of organic-inorganic hybrid beads from sepiolite and cellulose for effective adsorption of malachite green}

\author{
Xin Jiang, ${ }^{a}$ Sidun Wang, ${ }^{\mathrm{a}}$ Lan Ge, ${ }^{a}$ Fengcai Lin, ${ }^{a}$ Qilin Lu, ${ }^{a}$ Ting Wang, ${ }^{\mathrm{b}}$ Biao Huang ${ }^{\mathrm{a}}$ \\ and Beili Lu (ID *a
}

\begin{abstract}
Organic-inorganic hybrid adsorbents based on sepiolite and cellulose were prepared through an easy-tohandle procedure. Hydrogen-bonding existed between the silanol groups $(\mathrm{Si}-\mathrm{OH})$ on the sepiolite surface and the hydroxyl groups of the cellulose structure resulting in the formation of hybrid beads with good synergistic effects. Incorporation of the inorganic molecule sepiolite into the renewable polymer cellulose opened an opportunity for the development of alternative environment-friendly adsorbents with improved adsorption efficiency as well as enhanced thermal stability compared with neat cellulose beads. The utility of the obtained sepiolite/cellulose beads was demonstrated by investigating their performance for the removal of malachite green (MG). The maximum adsorption capacity of MG on sepiolite/cellulose beads was close to the calculated results from the Langmuir adsorption isotherm, and the adsorption kinetics followed well to the pseudo-second-order model.
\end{abstract}

\section{Introduction}

Hybrid materials composed of organic polymers and inorganic molecules have received much attention in diversified areas because of their unique properties. ${ }^{1}$ In this new class of materials, organic and inorganic components work synergistically to construct innovative composites with multi-functions and enhanced performance over conventional single-component materials. $^{2}$ The combination of the superior characteristics of organic polymers (toughness, flexibility and processability) and inorganic molecules (rigidity, heat resistance and good mechanical strength) is able to satisfy the requirements for a wide range of applications, such as catalyst carriers, ${ }^{3 a}$ electronics, ${ }^{3 b}$ adsorbents, ${ }^{3 c}$ energy storage ${ }^{3 d}$ and medicine. ${ }^{3 e}$

It is well known that adsorption is one of the most important techniques for the removal of chemically and biologically stable pollutants from wastewater, enjoying many advantages such as easy operation, high capacity and insensitivity of adsorbents. ${ }^{4}$ In view of this technique, the adsorbent is considered to be a key point for an efficient adsorption process. Although activated carbon is widely used as a conventional adsorbent, it is restricted in usage by the high cost and energy involved in preparation of the adsorbent. ${ }^{5}$ Therefore, a number of sustainable adsorbents have been designed and fabricated to meet the ever-increasing desire for practical applications.

${ }^{a}$ College of Material Engineering, Fujian Agriculture and Forestry University, Minhou County, Fuzhou City, Fujian Province 350108, China. E-mail: lubl@fafu.edu.cn

${ }^{b}$ Guangxi Key Laboratory of Chemistry and Engineering of Forest Products, Nanning City, Guangxi Province 530006, China
Organic-inorganic hybrid adsorbents, one of the most promising materials to replace conventional single-component adsorbents, have become an attractive option as they combined superior adsorption properties and improved structural stability. In addition, the performance of the hybrid adsorbents could be easily modified with the change of the components. However, the most commonly used organic polymers are obtained from fossil fuel which is liable to cause environmental pollution. The incorporation of renewable resource-based polymers into the hybrid adsorbents opened an opportunity for the development of an alternative environment-friendly adsorbents. ${ }^{6}$

Cellulose, one of the most abundant renewable polymers, has been successfully applied to deal with hazardous waste pollution (dyes, heavy metal ions) in the water. ${ }^{7}$ Its adsorption potential can be attributed to the hydrophilic and cationic properties of the large number of hydroxyl groups. Due to the limited adsorption performance of natural cellulose, ways of enhancing the adsorption ability of cellulose are in high demand, as they may improve its application scope. To date, there have been substantial efforts on the modification of cellulose to improve its adsorption performance. ${ }^{8}$ However, the production of modified cellulose usually involves harmful chemicals, multiple synthetic steps and a time-consuming purification procedure. In this context, assembly of cellulose with inorganic molecules may provide an alternative strategy for the construction of biodegradable, low-cost, non-toxic and mechanical stable hybrid adsorbents.

Sepiolite is a fibrous magnesium hydrosilicate with a unique pore structure composed of an alteration of blocks and 
channels extending in the fiber direction. ${ }^{9}$ Because of the large surface area, sepiolite has been proved to be a powerful adsorbent for the removal of waste pollutant, offering significant advantages like the abundance, easy availability and high adsorption capacity. ${ }^{10}$ The presence of numerous silanol groups at the surface of sepiolite allowed the building of strong interaction with other polymers, leading to improved properties of materials. ${ }^{\mathbf{1 1}}$

Most recently, materials made from sepiolite and organic biopolymers such as starch, ${ }^{12 a}$ chitosan $^{12 b}$ and poly-lactic acid ${ }^{12 c}$ have become an intensive topic from economical and environmental point of view. Nevertheless, in the case of cellulose, few studies on the use of composites based on cellulose and sepiolite as an adsorbent for the removal of waste pollutant, especially organic dyes, has been reported. ${ }^{13}$ Due to the powdered sepiolite and cellulose which may cause difficulty for the handling and filtration, separation of the adsorbents effectively needed to be addressed in large scale water treatment. As a particle shape of cellulose, cellulose-based beads facilitate the easy separation of hybrid adsorbents in batch operation. For example, Zhang et al. prepared a magnetic cellulose bead entrapping activated carbon to remove dyes including methylene blue and methyl orange. ${ }^{\mathbf{1 4}} \mathrm{Li}$ et al. successfully developed an adsorbent based on the maleic anhydride-modified cellulose beads and alkali-treated diatomite. ${ }^{15}$

Malachite green (MG, cationic dye) was one of the most widely used dyes in textile, distillery, paper, medical disinfectant and fish industries. ${ }^{\mathbf{1 6 a}}$ However, MG in wastewater is toxic to organisms and can cause environmental degradation. ${ }^{\mathbf{1 6 b}}$ Therefore, we proposed a facile, environmentally benign strategy to construct the hybrid beads derived from sepiolite and cellulose for the removal of MG from aqueous solutions. This approach utilizes readily available and non-toxic raw materials (bamboo pulp, sepiolite). Furthermore, the synthetic simplicity and ambient experimental conditions leads to industrial interest in the procedure. The morphology, structure, thermal stability and adsorption behaviors of the hybrid beads are also investigated to evaluate its potential application for the removal of organic dyes.

\section{Experimental}

\subsection{Materials}

Bamboo pulp ( $\alpha$-cellulose, $>95 \%$ ) was provided by Nanping Paper Co., Ltd. (Nanping, Fujian, China) using kraft pulping process followed by hypochlorite bleaching. Then, it was cut into pieces and beaten to form powdered cellulose with highefficient pulverizer. The molecular weight distribution of cellulose was analyzed by gel permeation chromatography (GPC) using a Waters 1515 isocratic HPLC pump with a Waters 2414 UV absorbance detector, and DMAc/LiCl was employed as an eluent. The weight average molecular weight $M_{\mathrm{w}}$ and the number average molecular weight $M_{\mathrm{n}}$ of cellulose was determined as $203 \mathrm{k}$ and $58 \mathrm{k}$, respectively. Malachite green (MG) was purchased from Aladdin Industrial Corporation (Shanghai, China) and used as received. Sepiolite was provided by Langfang Advanced Materials Co., Ltd. (Hebei, China). Sodium hydroxide $(\mathrm{NaOH})$, calcium chloride $\left(\mathrm{CaCl}_{2}\right)$, urea and hydrochloric acid $(\mathrm{HCl})$ were purchased from Sinopharm Chemical Reagent Beijing Co., Ltd. (Beijing, China). All reagents used in this work were of analytical grade, and deionized water was used for the preparation of aqueous solutions.

\subsection{Pretreatment of sepiolite}

The raw sepiolite was treated beforehand according to the following steps: $100 \mathrm{~g}$ sepiolite was added into $1000 \mathrm{~mL} \mathrm{15 \%}$ hydrochloric acid solutions with mechanically stirring for $48 \mathrm{~h}$ at room temperature. The obtained suspension was filtered and washed by deionized water. After that, it was introduced into planetary ball mill and milled at a rotational speed of $650 \mathrm{rpm}$ for four hours, followed by centrifuging and drying to get the samples for the further experiments.

\subsection{Preparation of sepiolite/cellulose hybrid bead (SCB)}

The powdered cellulose ( $4 \mathrm{~g}$ ) was dispersed in $7 \mathrm{wt} \% \mathrm{NaOH} / 12$ $\mathrm{wt} \%$ urea aqueous solution (100 g) which was pre-cooled to $-23{ }^{\circ} \mathrm{C}$ under vigorous stirring to obtain a homogeneous cellulose solution as procedures reported in the literature. ${ }^{\mathbf{1 7}}$ Then a certain amount of pretreated sepiolite was added into the above cellulose solution and mechanically stirred for 2 hours at room temperature. The resulting suspension was dropped through a $5 \mathrm{~mL}$ glass syringe at the dropping rate of 3 $\mathrm{mL} \min ^{-1}$ into $5 \mathrm{wt} \% \mathrm{HCl}$ solution containing $5 \mathrm{wt} \% \mathrm{CaCl}_{2}$, leading to well defined hydrogel beads. The obtained hydrogel beads were filtered and immersed in deionized water bath to remove residual un-participated ingredients, followed by freezedrying to get the sepiolite/cellulose hybrid beads (SCBs). The synthetic procedure was generalized in Fig. 1. SCB with $3: 4$ weight ratio of sepiolite and cellulose was labeled as SCB-34. The pure cellulose bead without sepiolite was fabricated by the same process and labeled as CB.

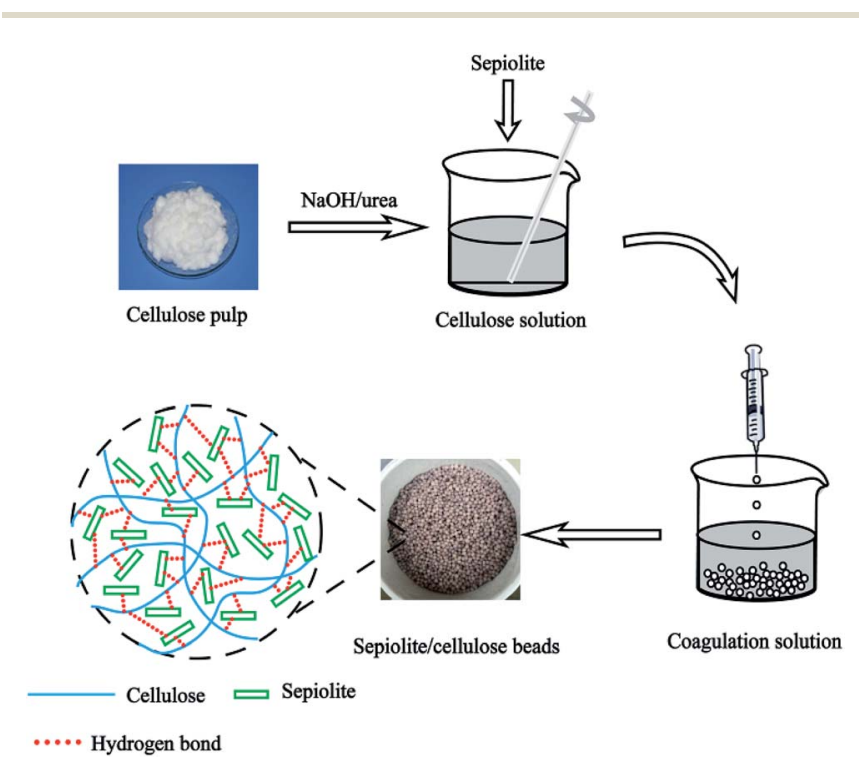

Fig. 1 Schematic illustration for the preparation of sepiolite/cellulose hybrid beads. 


\subsection{Characterization of the hybrid beads}

FTIR spectra of the samples were studied with a Nicolet 380 FTIR spectrometer (Thermo Electron Instruments Co., Ltd., USA) in the frequency range of $4000-400 \mathrm{~cm}^{-1}$ with a total of 32 scans and a resolution of $4 \mathrm{~cm}^{-1}$. Prior to analysis, each sample was first ground with $\mathrm{KBr}$ and pressed into thin pellets.

The sizes of $\mathrm{CB}$ and SCB-34 were analyzed by the SEM (SU8010 FE-SEM, Hitachi, Japan) images of the samples, ${ }^{18}$ which were further confirmed by the image analysis software (Image J) based on the digital photographs. ${ }^{19}$ The porosities of CB and SCB-34 were calculated by the following equations (eqn (1) and (2)): ${ }^{20}$

$$
\begin{gathered}
\text { Porosity }=\left(1-\frac{\rho_{\text {bead }}}{\rho_{\text {solid }}}\right) \times 100 \% \\
\rho_{\text {solid }}=\frac{M_{\text {sep }}+M_{\text {cel }}}{M_{\text {sep }} / \rho_{\text {sep }}+M_{\text {cel }} / \rho_{\text {cel }}}
\end{gathered}
$$

where $\rho_{\text {bead }}$ is the density of the bead (CB or SCB-34) determined according to the weight and volume of the samples; $\rho_{\text {solid }}$ is the density of the solid, which can be calculated according to eqn (2). $M_{\text {sep }}$ and $M_{\text {cel }}$ is the mass fraction of sepiolite and cellulose, respectively. $\rho_{\text {sep }}$ and $\rho_{\text {cel }}$ is the density of sepiolite and cellulose, respectively.

The surface and fracture surface morphology of samples were characterized with SU8010 FE-SEM (Hitachi, Japan) at an accelerating voltage of $1.0 \mathrm{kV}$. The thermal stability of the beads was characterized with a thermal gravimetric analyzer (NETZSCH STA 449 F3 Jupiter). The samples were heated from 25 to $800{ }^{\circ} \mathrm{C}$ at a heating rate of $10{ }^{\circ} \mathrm{C} \mathrm{min}^{-1}$ under nitrogen atmosphere with a flow rate of $25 \mathrm{~mL} \mathrm{~min}^{-1}$.

\subsection{Adsorption experiments}

The experiments for the adsorption of MG from aqueous solutions were carried out as follows: $10 \mathrm{mg}$ cellulose-based beads (CB and SCB) with different weight ratios of sepiolite to cellulose were added into a $50 \mathrm{~mL}$ conical flask containing $10 \mathrm{~mL}$ of MG solution. The resulting mixtures was shaken in a thermostat shaker (SHA-C, Kaihang Instrument Company, China) with a constant speed $(150 \mathrm{rpm})$ at $30{ }^{\circ} \mathrm{C}$. The effects of contact time, initial $\mathrm{pH}$ value and initial dye concentration on the adsorption behavior were also investigated respectively. The initial $\mathrm{pH}$ value was adjusted by adding drops of $0.1 \mathrm{M} \mathrm{NaOH}$ and/or $0.1 \mathrm{M}$ $\mathrm{HCl}$ solutions. All the conical flasks were sealed to avoid the evaporation of the water. The initial and residual MG concentrations were analyzed with UV spectrophotometer (Agilent8453, Agilent technologies, USA) at $618 \mathrm{~nm}$.

The dye removal efficiency $(R$, eqn (3)) and the adsorption capacity $\left(q_{\mathrm{e}}, \mathrm{mg} \mathrm{g}^{-1}\right.$, eqn (4)) were calculated as follows:

$$
\begin{gathered}
R(\%)=\frac{C_{0}-C_{\mathrm{e}}}{C_{0}} \times 100 \\
q_{\mathrm{e}}=\frac{C_{0}-C_{\mathrm{e}}}{m} \times V
\end{gathered}
$$

where $C_{0}\left(\mathrm{mg} \mathrm{L}^{-1}\right)$ and $C_{\mathrm{e}}\left(\mathrm{mg} \mathrm{L}^{-1}\right)$ are the initial and equilibrium concentration of $\mathrm{MG}$ in the solution, respectively; $V$ is the volume of MG solution (L) and $m$ is the weight of CB or SCB (g). The adsorption data at different time intervals were used to determine the kinetic parameters of MG adsorption. Moreover, the isotherms for adsorption were obtained by analyzing the adsorption capacity at different initial concentrations.

\section{Results and discussion}

\subsection{FTIR analysis}

FTIR spectra of the sepiolite, pure cellulose bead and sepiolite/ cellulose hybrid bead were presented in Fig. 2 to study changes of the material structure. As can be seen in Fig. 2, some characteristic peaks of sepiolite were identified: (i) the band at $3675 \mathrm{~cm}^{-1}$ was assigned to the hydroxyl stretching vibration of $\mathrm{Mg}-\mathrm{OH}$ group in octahedral layers, (ii) the appearance of peaks at $3407 \mathrm{~cm}^{-1}$ and $1636 \mathrm{~cm}^{-1}$ were due to the vibrations of structurally bound water or zeolitic water, (iii) the absorbance at $1091 \mathrm{~cm}^{-1}$ and $949 \mathrm{~cm}^{-1}$ were associated with the stretching of $\mathrm{Si}-\mathrm{O}$ bond, (iv) the peak at $1020 \mathrm{~cm}^{-1}$ could be attributed to the Si-O-Si plane vibration. ${ }^{21}$ As for pure cellulose bead, the characteristic peaks of cellulose could be observed at $3441 \mathrm{~cm}^{-1}$ (hydrogen bond $\mathrm{O}-\mathrm{H}$ stretching vibration), $2920 \mathrm{~cm}^{-1}$ (the $\mathrm{C}-\mathrm{H}$ symmetric stretching vibration), $1645 \mathrm{~cm}^{-1}(\mathrm{O}-\mathrm{H}$ bending of the absorbed water), $1065 \mathrm{~cm}^{-1}$ (the $\mathrm{C}-\mathrm{O}$ stretching vibration), and $895 \mathrm{~cm}^{-1}$ (the asymmetric out-of plane ring stretching). ${ }^{22}$ Compared with the spectra of $\mathrm{CB}$, the peak assigned to $\mathrm{O}-\mathrm{H}$ stretching shifted from $3441 \mathrm{~cm}^{-1}$ to $3421 \mathrm{~cm}^{-1}$ in the FTIR spectrum of SCB-34, presumably due to the hydrogen-bonding existed between the hydroxyl groups of cellulose structure and the silanol groups $(\mathrm{Si}-\mathrm{OH})$ on the sepiolite surface. ${ }^{23}$ Hence, the strong interactions between sepiolite and cellulose could be expected, resulting in the formation of hybrid beads with improved adsorption capacity and heat resistance, which would be demonstrated in the following experiments. In addition, the new peaks at 3672, 1020 and $947 \mathrm{~cm}^{-1}$ appeared in the spectra of SCB-34, further indicating the involvement of sepiolite in the hybrid beads.

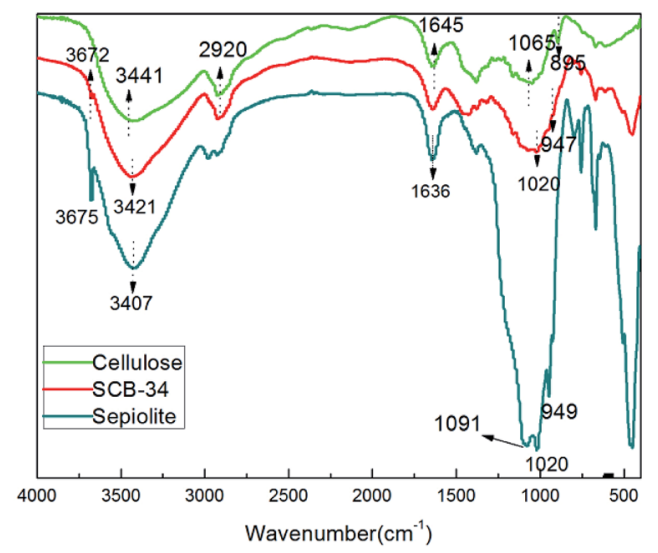

Fig. 2 FTIR spectra of sepiolite, pure cellulose bead (CB) and sepiolite/ cellulose hybrid bead with a weight ratio of $3: 4$ (SCB-34). 


\subsection{Size and porosity analysis}

In the digital photographs of CB and SCB-34, as shown in Fig. 3, all the samples exhibited well spherical shape. The SEM images determined that the mean diameter of $\mathrm{CB}$ and SCB-34 was $2.4 \mathrm{~mm}$ and $2.8 \mathrm{~mm}$, respectively. The larger size of SCB-34 was caused by the brace effect of sepiolite throughout the scaffold of cellulose-based bead. ${ }^{24}$ The densities and porosities of CB and SCB-34 have been calculated by eqn (1) and (2), and the results are summarized in Table 1 . Compared with CB, the porosity of SCB34 increased from $95.93 \%$ to $97.63 \%$ by incorporation of sepiolite, which contributed to the enhanced adsorption ability of SCB-34.

\subsection{Morphology analysis}

To get insight into the structure characteristics of our hybrid adsorbents, the morphologies of sepiolite, the surface and cross sections of pure cellulose bead and its hybrid bead with $3: 4$ weight ratio of sepiolite to cellulose (SCB-34) were investigated by field emission scanning electron microscopy (FESEM) respectively (Fig. 4). One can see that sepiolite presented rod and needle like morphology (Fig. 4a). In comparison with the surface morphology of CB, the surface of SCB-34 exhibited more folded and undulant structure, as a result of the incorporation of sepiolite into the cellulose bead, which is consistent with the FTIR observation (Fig. 4b and c). ${ }^{25}$ Fig. $4 \mathrm{~d}$ and e displayed the cross sections of CB and SCB-34 to reveal interior structure of the beads. A rough, interconnected and highly porous structure can be observed in the hybrid bead (SCB-34), contributed to the increasing active sites available for the dye adsorption, and led to improved adsorption capacities of hybrid beads. ${ }^{16 b}$

\subsection{Thermogravimetric analysis}

To clarify the thermal properties of sepiolite, pure cellulose bead and sepiolite/cellulose bead, their TGA and DTG curves were shown in Fig. 5. As for sepiolite, a total mass loss of $14.9 \%$ over the temperature range between $25{ }^{\circ} \mathrm{C}$ and $800{ }^{\circ} \mathrm{C}$ was observed in Fig. $5 \mathrm{a} .{ }^{26} \mathrm{CB}$ showed an initial weight loss of $10 \%$

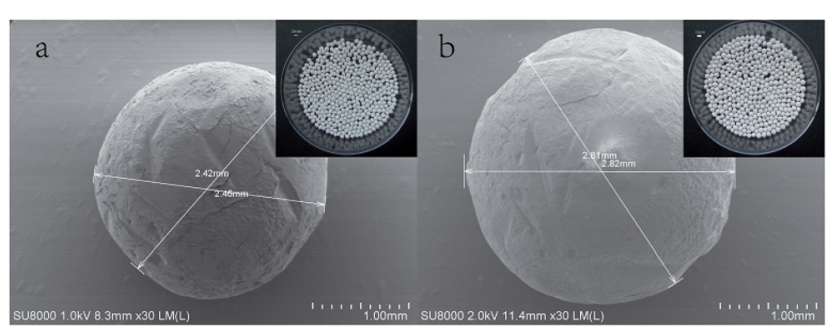

Fig. 3 SEM images of (a) CB, (b) SCB-34 (embedded of (a) and (b) present the corresponding digital photographs).

Table 1 Densities and porosities of CB and SCB-34

\begin{tabular}{llll}
\hline Sample & $\rho_{\text {bead }}\left(\mathrm{g} \mathrm{cm}^{-3}\right)$ & $\rho_{\text {solid }}\left(\mathrm{g} \mathrm{cm}^{-3}\right)$ & Porosity (\%) \\
\hline CB & 0.02063 & 0.5070 & $95.93 \%$ \\
SCB-34 & 0.02424 & 1.0209 & $97.63 \%$
\end{tabular}
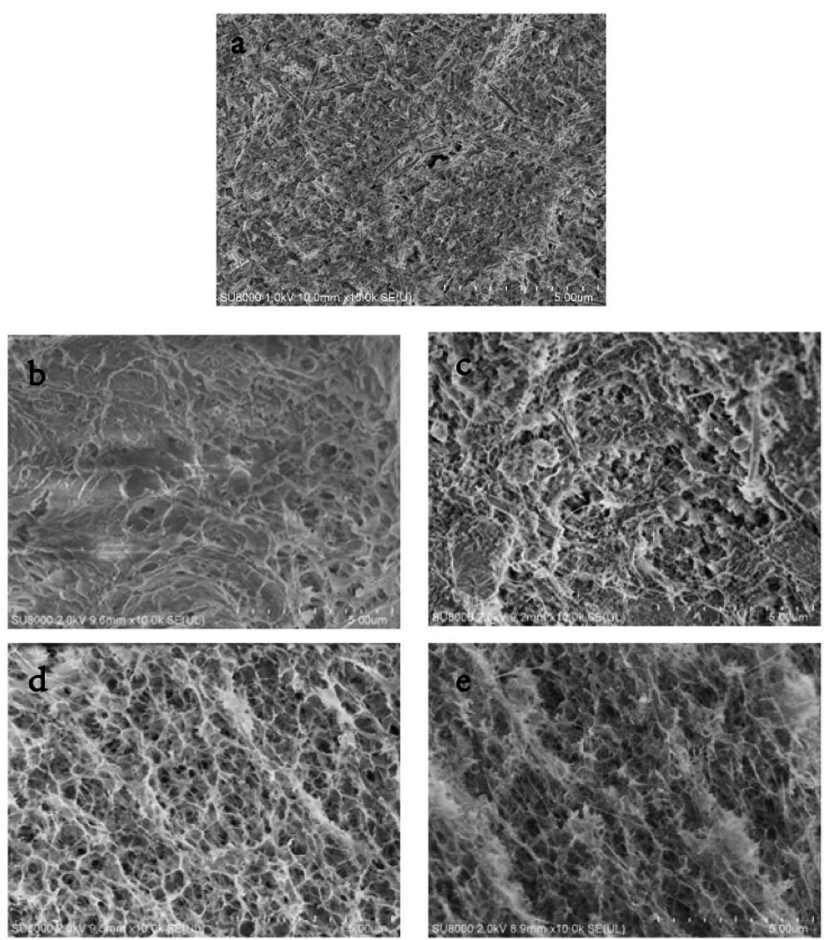

Fig. 4 Field emission scanning electron microscopy (FESEM) micrographs of (a) sepiolite, (b) the surface of CB and (c) SCB-34, (d) the fractured cross sections of $C B$ and (e) SCB-34.

between $25^{\circ} \mathrm{C}$ and $105^{\circ} \mathrm{C}$, which was caused by the evaporation of bound water from the sample. In the case of SCB-34, the release of water from sample caused less weight loss (5\%), presumably due to its more hydrophobic properties compared with $\mathrm{CB} .{ }^{27}$ Moreover, it can be seen that the incorporation of sepiolite into cellulose bead caused an increase in the initial thermal decomposition temperature from $309{ }^{\circ} \mathrm{C}$ to $320{ }^{\circ} \mathrm{C}$. From Fig. 5b, it was found that the thermal decomposition peak of the maximum weight loss is $347^{\circ} \mathrm{C}$ for SCB-34, which is $10{ }^{\circ} \mathrm{C}$ higher than in the case of $\mathrm{CB}$. Obviously, the hybrid beads showed higher heat resistant properties than pure cellulose bead, because of the confinement and thermal insulation effect of inorganic molecules sepiolite. ${ }^{28}$

\subsection{Effect of weight ratio of sepiolite to cellulose}

Assembly of sepiolite into cellulose can not only assign the thermal stability but also increase adsorption ability of the hybrid beads. Thus, the weight ratio of sepiolite to cellulose was considered as an important parameter in adsorption studies, and the effect of which on MG removal efficiency was shown in Fig. 6a. It was found that the removal efficiency of MG increased dramatically with the increase of the weight ratio of sepiolite to cellulose with an initial dye concentration of $50 \mathrm{mg} \mathrm{L}^{-1}$ at $30{ }^{\circ} \mathrm{C}$ for $3 \mathrm{~h}(\mathrm{pH}=7.0)$, reaching a maximum of $97.5 \%$ at $3: 4$ weight ratio. This phenomenon could be explained by the increase of the active sites available for MG with the increase of the amount of sepiolite. ${ }^{29}$ The percentage removal of MG decreased at higher weight ratio of sepiolite to cellulose $(4: 4)$, probably due to the collapse of pore structure caused by excessive amount of 

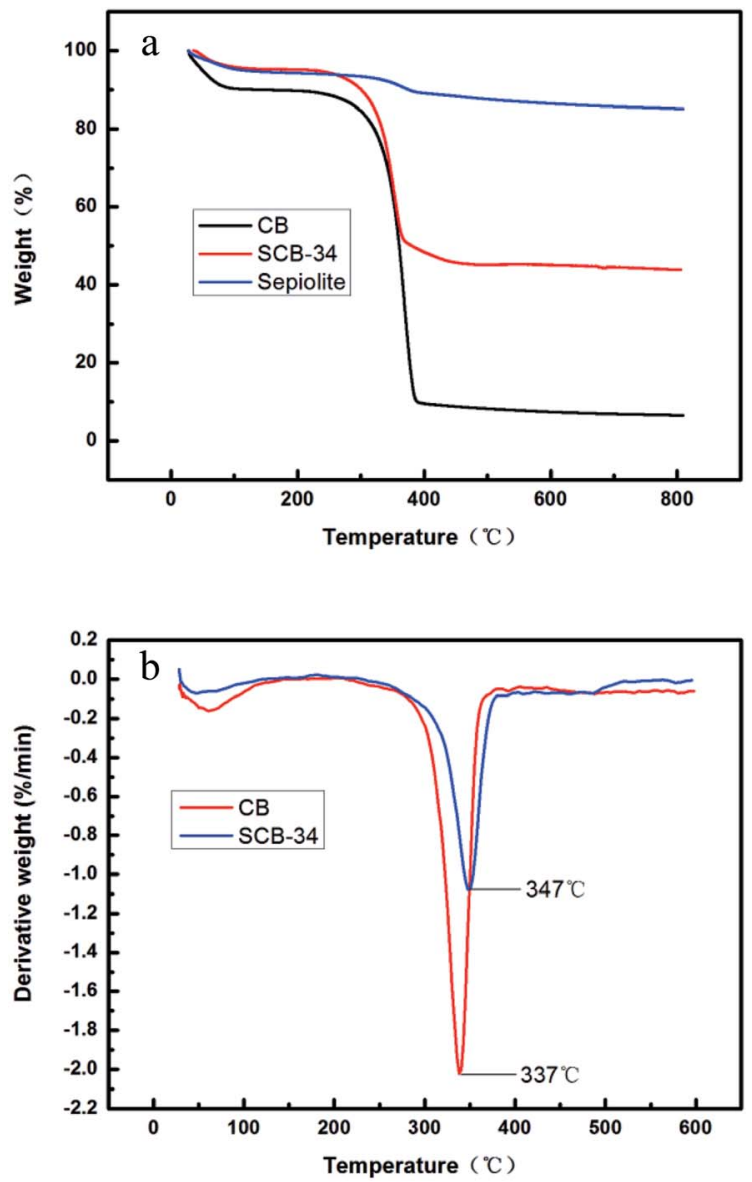

Fig. 5 (a) TGA curves of sepiolite, CB and SCB-34, (b) DTG curves of $\mathrm{CB}$ and SCB-34.

sepiolite. ${ }^{15}$ Therefore, $3: 4$ of sepiolite to cellulose was selected as the optimum weight ratio. As a comparison, the experiments for the removal of MG with cellulose bead and sepiolite were conducted separately. $11.0 \%$ and $73.5 \%$ of $\mathrm{MG}$ removal percentage was obtained, which is lower than that of SCB-34. These results demonstrate that by incorporation of sepiolite into cellulose, the adsorption efficiency of bio-based hybrid adsorbents was greatly improved.

\subsection{Effect of contact time}

Fig. 6b showed the effect of contact time on the dye removal efficiency of MG by changing the time range from $30 \mathrm{~min}$ to $300 \mathrm{~min}$. It can be seen that the removal of MG changed significantly from $44.6 \%$ to $91.6 \%$ in the first $180 \mathrm{~min}$. When the contact time was increased to $240 \mathrm{~min}$, the equilibrium removal efficiency of $97.6 \%$ could be reached. Inconspicuously influence on the adsorption efficiency was observed when further increasing the contact time. As a result, $240 \mathrm{~min}$ was the optimum contact time for the adsorption of MG by using hybrid beads (SCB-34) as the adsorbents.

\subsection{Effect of initial $\mathrm{pH}$ value}

As can be seen in Fig. 6c, the adsorption behavior of sepiolite/ cellulose hybrid beads for MG (cationic dye) was significantly affected by the initial $\mathrm{pH}$ value of solution, since it was the main factor to regulate the surface charge of SCBs. In general, the removal efficiency of $\mathrm{MG}$ is below $22.6 \%$ at a low $\mathrm{pH}$ value $(\mathrm{pH}<$ 2 ), possibly due to the electrostatic repulsion between adsorbent and MG caused by reducing of negatively charged adsorbent sites, meanwhile the competitive adsorption between excess $\mathrm{H}^{+}$and cationic $\mathrm{MG}$ on adsorbents had a negative effect on the removal efficiency. ${ }^{30}$ When $\mathrm{pH}$ value increased from 2 to 7, the removal of MG increased sharply to its maximum, which might be explained by the enhanced electrostatic interaction between cationic MG and negatively charged SCBs at a lower $\mathrm{H}^{+}$ concentration. ${ }^{31}$ As such, the optimum initial $\mathrm{pH}$ value for MG adsorption was found to be 7. If $\mathrm{pH}>7$, the adsorption of MG on SCBs dropped a little. The deprotonation of abundant $\mathrm{Si}-\mathrm{OH}$ group on the hybrid beads in alkaline conditions might influence the hydrogen bonding between dyes and adsorbents. ${ }^{32 a}$ On the other hand, the increase of solubility of the hybrid adsorbents at $\mathrm{pH}>7$ resulted in a little dissolution of adsorbents, which was not favorable for the removal of dyes. ${ }^{32 b}$

\subsection{Effect of initial dye concentration}

In addition, the adsorption capacity of MG with SCBs was investigated by using various initial dye concentrations (Fig. 6d). When the initial concentrations changed from 50 to $400 \mathrm{mg} \mathrm{L}^{-1}$, the adsorption capacity of MG increased quickly attributed to the enhanced driving force between MG and SCBs with a rise in the initial concentration. ${ }^{33}$ While, the adsorption capacity tended to level off at higher initial concentrations, implying the active sites of adsorbents reached saturation. ${ }^{30}$ Finally, the maximum adsorption capacity was found to be $382 \mathrm{mg} \mathrm{g}^{-1}$.

\subsection{Adsorption kinetics}

The adsorption kinetics was analyzed by employing pseudofirst-order and pseudo-second-order kinetic models to evaluate the adsorption process involved in the removal of MG by SCBs. The linear forms of pseudo-first-order and pseudosecond-order kinetic models are expressed in eqn (5) and (6), respectively. ${ }^{34}$

$$
\begin{gathered}
\ln \left(q_{\mathrm{e}}-q_{t}\right)=\ln q_{\mathrm{e}}-k_{1} t \\
\frac{t}{q_{t}}=\frac{1}{k_{2} q_{\mathrm{e}}^{2}}+\frac{t}{q_{\mathrm{e}}}
\end{gathered}
$$

where $q_{\mathrm{e}}\left(\mathrm{mg} \mathrm{g}^{-1}\right)$ is the adsorption capacity at equilibrium, and $q_{\mathrm{t}}\left(\mathrm{mg} \mathrm{g}^{-1}\right)$ is the adsorption capacity at time $t$ (min). The parameters $k_{1}(1 / \mathrm{min})$ and $k_{2}\left(\mathrm{~g} \mathrm{mg}^{-1} \mathrm{~min}^{-1}\right)$ are the rate constants of the pseudo-first-order and pseudo-second-order kinetic models, respectively.

The plots according to the pseudo-first-order and pseudosecond-order models are shown in Fig. 7. Moreover, the values of kinetic parameters including the calculated equilibrium adsorption capacity $\left(q_{\mathrm{e}}\right)$, the rate constants $\left(k_{1}, k_{2}\right)$ and the correlation coefficients $\left(R^{2}\right)$ are summarized in Table 2 . A good linearity was observed in the plot of $t / q_{t}$ against $t$, which was further confirmed by the high correlation coefficient 

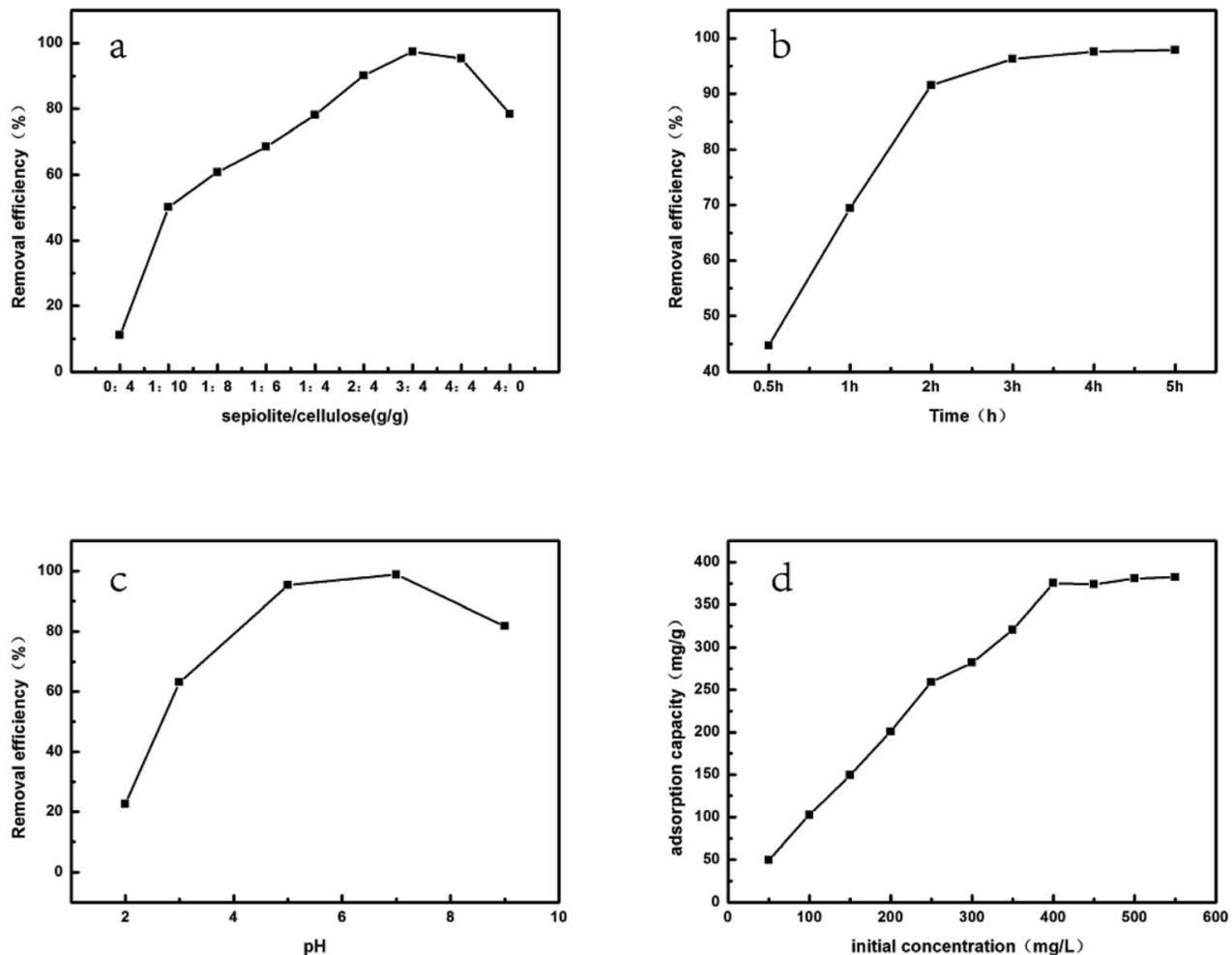

Fig. 6 (a) Effect of weight ratio of sepiolite to cellulose on the adsorption of MG, (b) effect of contact time on the adsorption of MG (weight ratio of sepiolite to cellulose $=3: 4$, initial dye concentration $=50 \mathrm{mg} \mathrm{L}^{-1}, \mathrm{pH}=7.0$ ), (c) effect of initial pH value on the adsorption of $\mathrm{MG}$ (weight ratio of sepiolite to cellulose $=3: 4$, initial dye concentration $=50 \mathrm{mg} \mathrm{L}^{-1}, t=240 \mathrm{~min}$ ), (d) effect of initial dye concentration on the adsorption of $\mathrm{MG}$ (weight ratio of sepiolite to cellulose $=3: 4, t=240 \mathrm{~min}, \mathrm{pH}=7.0$ ).
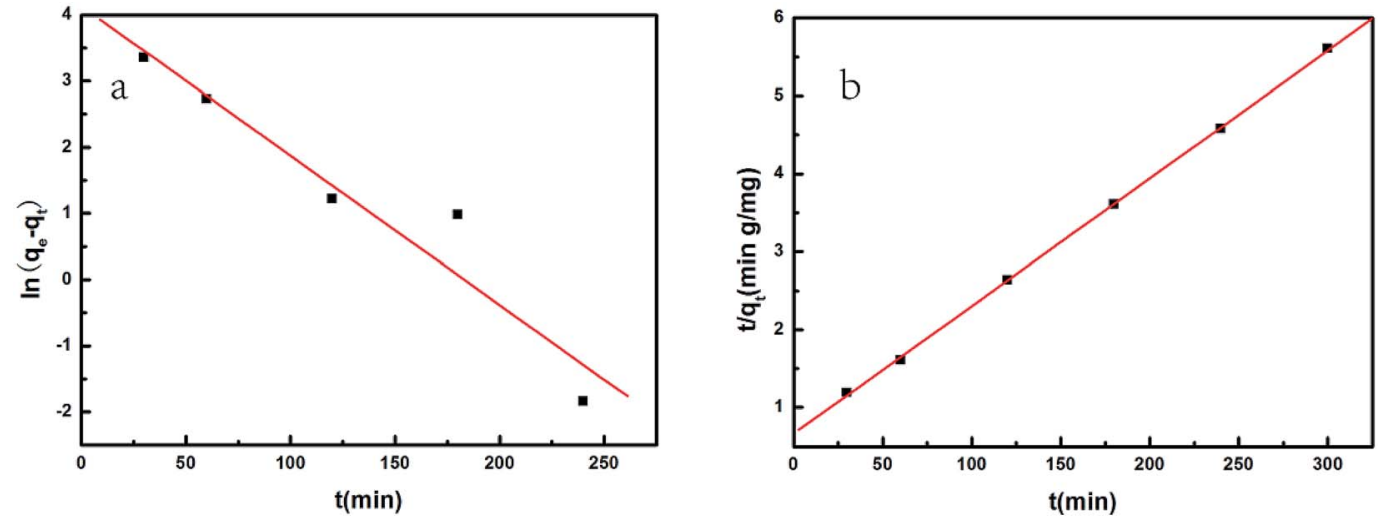

Fig. 7 (a) Pseudo-first-order and (b) pseudo-second-order plots for adsorption of MG on SCB-34.

$\left(R^{2}=0.9998\right)$ determined by the pseudo-second-order model. Also, the calculated equilibrium adsorption capacity $q_{\mathrm{e}}$ based on the pseudo-second-order model was found to be $61 \mathrm{mg} \mathrm{g}^{-1}$,

Table 2 The adsorption parameters of pseudo-first-order and pseudo-second-order kinetic models

\begin{tabular}{lllll}
\hline Kinetics models & $\begin{array}{l}q_{\mathrm{e}} \\
\left(\mathrm{mg} \mathrm{g}^{-1}\right)\end{array}$ & $\begin{array}{l}k_{1} \\
(1 / \mathrm{min})\end{array}$ & $\begin{array}{l}k_{2} \\
\left(\mathrm{~g} \mathrm{mg}^{-1} \mathrm{~min}^{-1}\right)\end{array}$ & $R^{2}$ \\
\hline Pseudo-first-order & 62.34 & 0.0226 & - & 0.9268 \\
Pseudo-second-order & 60.98 & - & 0.02476 & 0.9998
\end{tabular}

which is close to the experimental data. These results revealed that the adsorption process of MG onto SCBs fitted well with the pseudo-second-order kinetic model, suggesting that the overall rate of adsorption process was controlled by chemisorptions. $^{35}$

\subsection{Adsorption isotherm}

Furthermore, the Langmuir and Freundlich adsorption isotherms were applied to analyze the adsorption of MG on SCBs and to fit the experimental data. Eqn (7) and (8) showed the linear forms of these two models, respectively. ${ }^{36}$ 

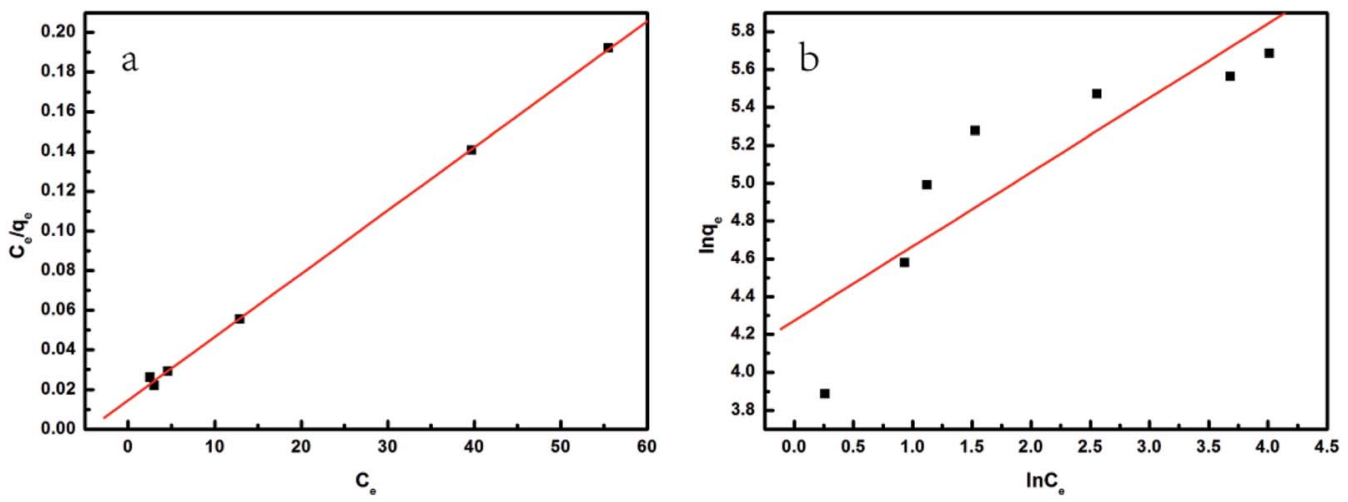

Fig. 8 (a) Plot of Langmuir isotherm, and (b) plot of Freundlich isotherm for the removal of MG using SCB-34 as adsorbents.

$$
\begin{gathered}
\frac{C_{\mathrm{e}}}{q_{\mathrm{e}}}=\frac{1}{b q_{\max }}+\frac{C_{\mathrm{e}}}{q_{\max }} \\
\ln q_{\mathrm{e}}=\ln K_{\mathrm{F}}+\frac{1}{n_{\mathrm{F}}} \ln C_{\mathrm{e}}
\end{gathered}
$$

where $q_{\mathrm{e}}\left(\mathrm{mg} \mathrm{g}^{-1}\right)$ and $q_{\max }\left(\mathrm{mg} \mathrm{g}^{-1}\right)$ are the adsorption capacity at equilibrium and the maximum adsorption capacity, respectively. $C_{\mathrm{e}}\left(\mathrm{mg} \mathrm{L}^{-1}\right)$ is the equilibrium concentration; $b\left(\mathrm{~L} \mathrm{mg}^{-1}\right)$ is the Langmuir constant. $K_{\mathrm{F}}\left(\mathrm{L} \mathrm{mg}^{-1}\right)$ and $n_{\mathrm{F}}$ are the Freundlich constants reflecting the adsorption capacity and adsorption intensity, respectively.

The linearized curves and the related parameters from Langmuir and Freundlich isotherm models are shown in Fig. 8 and Table 3, respectively. It was found that the adsorption behavior of MG on SCBs fitted well with the Langmuir model because of the much higher correlation coefficient value $\left(R^{2}=0.9993\right)$ compared with that of the Freundlich model. Therefore, in our systems, MG was adsorbed on the SCBs' surface through monolayer adsorption, resulting in a saturated adsorption capacity. ${ }^{19}$ It was noted that the $q_{\text {max }}$ value of MG on SCB was calculated as $314 \mathrm{mg} \mathrm{g}^{-1}$, which was compared to the maximum adsorption capacities of some other low-cost adsorbents including activated carbon (Table 4). It was found that SCB showed a relatively higher $q_{\max }$ value than other adsorbents reported previously. In addition, the value of $b$ was in the range 0 to 1 . As a result, MG could be easily adsorbed on the hybrid adsorbents (SCBs) prepared here exhibiting good adsorption capacity.

\subsection{Adsorption for other pollutants}

Additionally, the feasibility of the hybrid adsorbent (SCB-34) was verified by investigating its adsorption ability for a few

Table 3 The adsorption parameters of Langmuir and Freundlich isotherm models

\begin{tabular}{llllll}
\hline $\begin{array}{l}\text { Isotherm } \\
\text { models }\end{array}$ & $\begin{array}{l}q_{\max } \\
\left(\mathrm{mg} \mathrm{g}^{-1}\right)\end{array}$ & $\begin{array}{l}b \\
\left(\mathrm{~L} \mathrm{mg}^{-1}\right)\end{array}$ & $\begin{array}{l}K_{\mathrm{F}} \\
\left(\mathrm{L} \mathrm{mg}^{-1}\right)\end{array}$ & $n_{\mathrm{F}}$ & $R^{2}$ \\
\hline $\begin{array}{l}\text { Langmuir } \\
\text { Freundlich }\end{array}$ & 314.47 & 0.2072 & - & - & 0.9993 \\
& - & - & 71.77 & 2.55 & 0.7697
\end{tabular}

Table 4 Comparison of the maximum adsorption capacities of MG using different adsorbents

\begin{tabular}{lll}
\hline & $\begin{array}{l}q_{\max } \\
\left(\mathrm{mg} \mathrm{g}^{-1}\right)\end{array}$ & Reference \\
\hline Adsorbent & 0.180 & 37 \\
Bentonite clay & 7.72 & 38 \\
Graphene oxide/cellulose bead (GOCB) & 30.09 & 18 \\
Jute fiber carbon & 136.6 & 39 \\
Bagasse fly ash & 170.33 & 40 \\
Treated ginger waste & 188.6 & 41 \\
Cattail biomass based activated carbon & 210.18 & 42 \\
Sepiolite/cellulose hybrid bead (SCB) & 314.47 & This study
\end{tabular}

more pollutants, including methyl violet, $\mathrm{Zn(II),} \mathrm{Cd(II)} \mathrm{and} \mathrm{2,4-}$ dichlorophenol. As for methyl violet, the maximum adsorption capacity was found to be $177 \mathrm{mg} \mathrm{g}^{-1}$ with an initial dye concentration of $300 \mathrm{mg} \mathrm{L}^{-1}$ at $30{ }^{\circ} \mathrm{C}$ for $3 \mathrm{~h}(\mathrm{pH}=7.0)$. SCB-34 was also suitable to remove heavy metal ions, such as $\mathrm{Zn}$ (II) and Cd(II) from aqueous solutions. The maximum adsorption capacity was found to be $145 \mathrm{mg} \mathrm{g}^{-1}$ and $140 \mathrm{mg} \mathrm{g}^{-1}$, respectively (initial concentration $=250 \mathrm{mg} \mathrm{g}^{-1}, \mathrm{pH}=6.5, t=2 \mathrm{~h}$ ). In the case of 2,4-dichlorophenol, $35.7 \mathrm{mg} \mathrm{g}^{-1}$ of adsorption capacity can be achieved by using $100 \mathrm{mg} \mathrm{L}^{-1}$ of 2,4-dichlorophenol solutions at $30^{\circ} \mathrm{C}$ for $3 \mathrm{~h}(\mathrm{pH}=5.0)$.

\section{Conclusions}

In summary, an easily operated and environmentally-friendly procedure was developed to prepare sepiolite/cellulose hybrid beads as adsorbents under mild conditions. This approach utilizes readily available bamboo pulp and sepiolite as raw materials and features synthetic simplicity. Introduction of inorganic molecule sepiolite into organic cellulose not only significantly enhanced the adsorption efficiency but also facilitated the improvement of the thermal stability of neat cellulose bead. The abundant hydroxyl groups of cellulose, large specific surface area of sepiolite as well as the synergistic effect between these two components are believed to be the key factors for the superior performance of the hybrid adsorbents. Thus, SCBs are 
expected to be an alternative efficient adsorbent for the treatment of organic pollutants.

\section{Acknowledgements}

We acknowledge the generous financial support of the National Natural Science Foundation of China (No. 21402027), the Natural Science Foundation of Fujian Province (No. 2015J05046), open fund of Guangxi Key Laboratory of Chemistry and Engineering of Forest Products (No. GXFC 14-03) and Chemicals and Science Foundation for Distinguished Young Scholars of Fujian Agriculture and Forestry University (No. xjq201503).

\section{Notes and references}

1 C. Sanchez, P. Belleville, M. Popall and L. Nicole, Chem. Soc. Rev., 2011, 40, 696-753.

2 P. Gomez-Romero, Adv. Mater., 2001, 13, 163-174.

3 (a) M. H. Valkenberg and W. F. Hölderich, Catal. Rev.: Sci. Eng., 2002, 44, 321-374; (b) P. Reiss, E. Couderc, J. De Girolamo and A. Pron, Nanoscale, 2011, 3, 446-489; (c) B. Samiey, C.-H. Cheng and J. Wu, Materials, 2014, 7, 673726; (d) P. Gómez-Romero, O. Ayyad, J. Suárez-Guevara and D. Muñoz-Rojas, J. Solid State Electrochem., 2010, 14, 19391945; (e) P. Fattahi, G. Yang, G. Kim and M. R. Abidian, Adv. Mater., 2014, 26, 1846-1885.

4 I. Ali, Chem. Rev., 2012, 112, 5073-5091.

5 (a) G. Crini, Bioresour. Technol., 2006, 97, 1061-1085; (b) M. Rafatullah, O. Sulaiman, R. Hashim and A. Ahmad, J. Hazard. Mater., 2010, 177, 70-80.

6 D. Mohan, A. Sarswat, Y. S. Ok and C. U. Pittman Jr, Bioresour. Technol., 2014, 160, 191-202.

7 G. Annadurai, R.-S. Juang and D.-J. Lee, J. Hazard. Mater., 2002, 92, 263-274.

8 S. Hokkanen, A. Bhatnagar and M. Sillanpää, Water Res., 2016, 91, 156-173.

9 E. Ruiz-Hitzky, J. Mater. Chem., 2001, 11, 86-91.

10 Y. Özdemir, M. Doğan and M. Alkan, Microporous Mesoporous Mater., 2006, 96, 419-427.

11 G. Tartaglione, D. Tabuani, G. Camino and M. Moisio, Compos. Sci. Technol., 2008, 68, 451-460.

12 (a) M. Darder, C. R. S. Matos, P. Aranda, R. F. Gouveia and E. Ruiz-Hitzky, Carbohydr. Polym., 2017, 157, 1933-1939; (b) M. Darder, M. López-Blanco, P. Aranda, A. J. Aznar, J. Bravo and E. Ruiz-Hitzky, Chem. Mater., 2006, 18, 16021610; (c) K. Fukushima, D. Tabuani and G. Camino, Mater. Sci. Eng., C, 2012, 32, 1790-1795.

13 M. I. Carretero, J. M. Bernabé and E. Galán, Appl. Clay Sci., 2006, 33, 43-51.

14 X. Luo and L. Zhang, J. Hazard. Mater., 2009, 171, 340-347.

15 Y. Li, H. Xiao, M. Chen, Z. Song and Y. Zhao, J. Mater. Sci., 2014, 49, 6696-6704.

16 (a) B. H. Hameed and M. I. El-Khaiary, J. Hazard. Mater., 2008, 153, 701-708; (b) S. Srivastava, R. Sinha and D. Roy, Aquat. Toxicol., 2004, 66, 319-329.
17 C. Chang, L. Zhang, J. Zhou, L. Zhang and J. F. Kennedy, Carbohydr. Polym., 2010, 82, 122-127.

18 X. Zhang, H. Yu, H. Yang, Y. Wan, H. Hu, Z. Zhai and J. Qin, J. Colloid Interface Sci., 2015, 437, 277-282.

19 N. Mohammed, N. Grishkewich, R. M. Berry and K. C. Tam, Cellulose, 2015, 22, 3725-3738.

20 D. Killeen, M. Frydrych and B. Chen, Mater. Sci. Eng., C, 2012, 32, 749-757.

21 E. Eren, O. Cubuk, H. Ciftci, B. Eren and B. Caglar, Desalination, 2010, 252, 88-96.

22 J. Lu, P. Askeland and L. T. Drzal, Polymer, 2008, 49, 12851296.

23 M. Soheilmoghaddam, M. U. Wahit, A. A. Yussuf, M. A. AlSaleh and W. T. Whye, Polym. Test., 2014, 33, 121-130.

24 C. Deng, J. Liu, W. Zhou, Y.-K. Zhang, K.-F. Du and Z.-M. Zhao, Chem. Eng. J., 2012, 200-202, 452-458.

25 S. Leguey, A. I. Ruiz, R. Fernández and J. Cuevas, Am. J. Sci., 2014, 314, 1041-1063.

26 F. Chivrac, E. Pollet, M. Schmutz and L. Avérous, Carbohydr. Polym., 2010, 80, 145-153.

27 Q. Lu, X. Li, L. Tang, B. Lu and B. Huang, RSC Adv., 2015, 5, 56198-56204.

28 Z. Sárossy, T. O. J. Blomfeldt, M. S. Hedenqvist, C. B. Koch, S. S. Ray and D. Plackett, ACS Appl. Mater. Interfaces, 2012, 4, 3378-3386.

29 J. Tan, X. Zhang, X. Wei and L. Wang, BioResources, 2012, 7, 4307-4320.

30 N. Buvaneswari and C. Kannan, J. Hazard. Mater., 2011, 189, 294-300.

31 L. Liu, Z. Y. Gao, X. P. Su, X. Chen, L. Jiang and J. M. Yao, ACS Sustainable Chem. Eng., 2015, 3, 432-442.

32 (a) G. Kiani, M. Dostali, A. Rostami and A. R. Khataee, Appl. Clay Sci., 2011, 54, 34-39; (b) Z. Wu, I.-S. Ahn, C.-H. Lee, J.-H. Kim, Y. G. Shul and K. Lee, Colloids Surf., A, 2004, 240, 157-164.

33 H. Qiao, Y. Zhou, F. Yu, E. Wang, Y. Min, Q. Huang, L. Pang and T. Ma, Chemosphere, 2015, 141, 297-303.

34 M. Min, L. Shen, G. Hong, M. Zhu, Y. Zhang, X. Wang, Y. Chen and B. S. Hsiao, Chem. Eng. J., 2012, 197, 88-100.

35 Q. Peng, M. Liu, J. Zheng and C. Zhou, Microporous Mesoporous Mater., 2015, 201, 190-201.

36 Y. Zhou, X. Wang, M. Zhang, Q. Jin, B. Gao and T. Ma, Cellulose, 2014, 21, 2797-2809.

37 M. J. Iqbal and M. N. Ashiq, J. Hazard. Mater., 2007, 139, 5766.

38 S. S. Tahir and N. Rauf, Chemosphere, 2006, 63, 1842-1848.

39 K. Porkodi and K. Vasanth Kumar, J. Hazard. Mater., 2007, 143, 311-327.

40 I. D. Mall, V. C. Srivastava, N. K. Agarwal and I. M. Mishra, Colloids Surf., A, 2005, 264, 17-28.

41 R. Ahmad and R. Kumar, J. Environ. Manage., 2010, 91, 10321038.

42 M. Yu, Y. Han, J. Li and L. Wang, Chem. Eng. J., 2017, 317, 493-502. 